\title{
Transport along Null Curves
}

\author{
Joseph Samuel and Rajaram Nityananda \\ Raman Research Institute, Bangalore 560 080, INDIA.
}

email:sam@rri.ernet.in, rajaram@rri.ernet.in 


\begin{abstract}
Fermi Transport is useful for describing the behaviour of spins or gyroscopes following nongeodesic, timelike world lines. However, Fermi Transport breaks down for null world lines. We introduce a transport law for polarisation vectors along non-geodesic null curves. We show how this law emerges naturally from the geometry of null directions by comparing polarisation vectors associated with two distinct null directions. We then give a spinorial treatment of this topic and make contact with the geometric phase of quantum mechanics. There are two significant differences between the null and timelike cases. In the null case (i) The transport law does not approach a unique smooth limit as the null curve approaches a null geodesic. (ii) The transport law for vectors is integrable, i.e the result depends only on the local properties of the curve and not on the entire path taken. However, the transport of spinors is not integrable: there is a global sign of topological origin.
\end{abstract}




\section{Introduction}

The spin four-vector $s^{a}$ of a gyroscope (not acted on by external torques) moving along a timelike geodesic is parallel transported along the geodesic [1]. Similarly, the polarisation vector $v^{a}$ of a light ray following a null geodesic is parallel transported along the null geodesic. If a gyroscope follows a timelike curve which is not a geodesic, the parallel transport rule no longer applies: parallel transport does not preserve the orthogonality of the spin four-vector to the tangent vector for non-geodesic curves. The correct transport law for spin four-vectors along timelike curves is Fermi transport[1], 2]. However, Fermi transport does not apply to null curves: the transport equation breaks down. What is the appropriate transport law for polarisation vectors along null, non-geodesic curves? The purpose of this paper is to answer this question.

We will start in section II with a review of Fermi transport and introduce in section III a new transport law for null, non-geodesic curves and describe its properties. We then show in section IV that this law derives naturally from the geometry of null vectors just as Fermi transport derives naturally from the geometry of timelike vectors. Section V is a more sophisticated spinorial discussion of the transport law. Section VI connects the spinorial discussion of Section V to the geometric phase of a two state quantum system. Section VII is a concluding discussion.

\section{Review of Fermi Transport}

Let $(\mathcal{M}, g)$ be a space-time manifold with a Lorentzian metric $g$ of signature $(+,-,-,-)$. Let $\mathcal{C}$ be a smooth time-like curve in $\mathcal{M}$ and $p$ a point on the curve. In local coordinates, the curve is described as $x^{a}(\tau)$, where $\tau$ is an arbitrary parameter which increases into

the future. We define the tangent vector $\mathbf{t}$ by $t^{a}=d x^{a} / d \tau$ and the acceleration $\dot{\mathbf{t}}$ by $\dot{t}^{a}=t^{b} \nabla_{b} t^{a}$, where $\nabla_{b}$ is the covariant derivative. If $s^{a}$ is a vector at $p$ which is orthogonal to $\mathbf{t}\left(\mathbf{s} \in T_{p} \mathcal{M},(\mathbf{s} \cdot \mathbf{t})_{p}=0\right)$, Fermi transport gives us a vector at every point of $\mathcal{C}$ defined by the transport law

$$
D s^{a} / d \tau=F^{a b} s_{b}
$$

where

$$
F^{a b}=\frac{\left(\dot{t}^{a} t^{b}-t^{a} \dot{t}^{b}\right)}{t^{c} \cdot t_{c}}
$$


or more abstractly, $\mathbf{F}=\dot{\mathbf{t}} \wedge \mathbf{t} /(\mathbf{t} \cdot \mathbf{t})$. The transport law (1) has the following properties:

1. Vectors orthogonal to the tangent vector at $p$ are transported to vectors orthogonal to the tangent vector at other points of $\mathcal{C}$.

2. The transport law is covariant under reparametrization of the curve. If $\tau$ is changed to $\tau^{\prime}$, where $f:=d \tau / d \tau^{\prime}>0, \mathbf{t}$ and $\dot{\mathbf{t}}$ transform as follows

$$
\mathbf{t} \rightarrow f \mathbf{t} \quad \dot{\mathbf{t}} \rightarrow f^{2} \dot{\mathbf{t}}+\alpha_{1} \mathbf{t},
$$

where $\alpha_{1}$ is some function on $\mathcal{C}$.

As a result $\mathbf{F} \rightarrow f \mathbf{F}$ and so the vector field defined on $\mathcal{C}$ by Fermi transport does not depend on the parametrization of $\mathcal{C}$.

3. Inner products between vectors $\mathbf{s}_{1}$ and $\mathbf{s}_{2}$ are maintained under Fermi transport

$$
\frac{d\left(\mathbf{s}_{1} \cdot \mathbf{s}_{2}\right)}{d \tau}=\frac{D\left(\mathbf{s}_{1} \cdot \mathbf{s}_{2}\right)}{d \tau}=F^{a b} s_{1 a} s_{2 b}+F^{a b} s_{1 b} s_{2 a}=0
$$

from the antisymmetry of $F^{a b}$. In particular, an orthonormal triad $\left(\mathbf{e}_{1}, \mathbf{e}_{2}, \mathbf{e}_{3}\right)_{p}$ of vectors orthogonal to $\mathbf{t}$ at $p$ can be Fermi transported along $\mathcal{C}$ to give an orthonormal triad orthogonal to $\mathbf{t}$ everywhere on $\mathcal{C}$.

4. For geodesics Fermi transport reduces to parallel transport, since $\mathbf{F}$ vanishes on geodesics.

Although the treatment given above is in the context of an arbitrary curved spacetime, the notion of Fermi transport has nothing to do with curvature.This is evident because the entire discussion takes place in the neighborhood of a single open curve. By an appropriate choice of co-ordinates, all the Christoffel symbols can be made to vanish on this curve [3]. The curvature of space-time thus plays no essential role in the discussion of Fermi transport along an open curve, which should be viewed as a special relativistic kinematic effect. It is thus possible to conduct the whole discussion in flat space-time. By parallel transport we can identify the tangent space at any point $p^{\prime}$ along the curve $\mathcal{C}$ with $T_{p} \mathcal{M}$. The subsequent discussion is hence entirely within $T_{p} \mathcal{M}$.

Fermi transport arises from the geometry of timelike vectors in $T_{p} \mathcal{M}$. For a nongeodesic curve the vector field obtained by parallel transport of the tangent vector at one 
point does not agree with the local tangent vector at other points. Fermi transport allows us to set up a correspondence between vectors orthogonal to distinct timelike vectors, viz the tangent vectors at different points of a non-geodesic curve. This correspondence is geometrically natural but is not integrable. By integrability we mean that the result of transporting a given initial vector to a final point along the curve depens only on the local properties of the curve at the final point, and not on the entire path upto that point. In this sense, the Fermi transport rule is not integrable. This reflects the curvature of the space of timelike directions, which can be identified with the unit hyperboloid [3, 国 in Minkowski space.

\section{Transport along Null Non-Geodesics}

As is evident from (2), the Fermi transport rule breaks down for null curves, since the denominator of (2) vanishes. For geodesic null curves, polarisation vectors are parallel transported along the curve. But what about non-geodesic null curves? This is the question that we address and answer in this paper. In this section we will simply write down a transport law for polarisation vectors along null, non-geodesic curves. We will then show that this law has geometrically natural properties as does Fermi transport.

Let $\mathcal{N}$ be a smooth null curve described in local coordinates by $x^{a}(\tau)$, where $\tau$ is an arbitrary parameter which increases into the future. We use $l^{a}=d x^{a} / d \tau$ to denote the tangent vector to the null curve. We will also need $i^{a}=l^{b} \nabla_{b} l^{a}$ and $\ddot{l}^{a}=l^{b} \nabla_{b} i^{a}$. We will assume that $\mathcal{N}$ is nowhere geodesic i.e, $\mathbf{i}$ is nowhere a scalar multiple of $\mathbf{l}$. By differentiating $\mathbf{l} . \mathbf{l}=0$ with respect to $\tau$, we deduce in succession $\mathbf{l} . \mathbf{i}=0$ and $\mathbf{i} . \mathbf{i}+\mathbf{l} . \mathbf{i}=0$. Let us write $H_{p} \subset T_{p} \mathcal{M}$ for the space of all vectors in $T_{p} \mathcal{M}$ which are orthogonal to 1 at $p . H_{p}$ also includes $\mathbf{l}$, since $\mathbf{l}$ is null. The Lorentz metric at $p$, pulled back to $H_{p}$, has signature $(0,-,-)$. We need to "mod out" by the null direction $l^{a}$ to get a non-degenerate metric. We define a polarization vector to be an equivalence class of vectors in $H_{p}$ which differ by a multiple of $\mathbf{l}\left(\mathbf{v}_{1} \sim \mathbf{v}_{2} \Longleftrightarrow \mathbf{v}_{2}=\mathbf{v}_{1}+\lambda \mathbf{l}\right.$ for some $\left.\lambda\right)$. Our transport law will tell us how to transport polarization vectors along $\mathcal{N}$. (This "modding out" is standard for null curves [5]).

Given a polarisation vector (an equivalence class) at $p$, let us take a representative element $v^{a}$ from $H_{p}$. We transport this vector along $\mathcal{N}$ using the rule 


$$
\frac{D v^{a}}{D \tau}=K^{a b} v_{b}
$$

where $K^{a b}$ is an antisymmetric tensor defined by

$$
K^{a b}=\frac{\left(\ddot{l}^{a} \dot{l}^{b}-\dot{l}^{a} \ddot{l}^{b}\right)}{\left(\dot{l^{c}} \cdot \dot{l}_{c}\right)} .
$$

Finally we evaluate the equivalence class of $v^{a}\left(\bmod\right.$ out by $\left.l^{a}\right)$ to produce a polarisation vector field along $\mathcal{N}$.

This law, designated $K$ transport from now on, has the following properties:

1. It is straightforward to check that polarization vectors (equivalence classes of vectors perpendicular to l) at $p$ are transported to polarization vectors at other points of $\mathcal{N}$.

2. The transport law is reparametrization covariant: Under reparametrization of $\mathcal{N}$, $1 \rightarrow f 1$ and

$$
\mathrm{i} \rightarrow f^{2} \mathbf{i}+\alpha_{2} \mathbf{l}, \quad \ddot{\mathbf{i}} \rightarrow f^{3} \ddot{\mathbf{l}}+\alpha_{3} \dot{\mathbf{i}}+\alpha_{4} \mathbf{l},
$$

where the $\alpha$ 's are some functions on $\mathcal{N}$. Using (7) in (5).6), we can drop the $\alpha_{2}$ and $\alpha_{4}$ terms, which are proportional to 1 : they either vanish when they are contracted with $v_{b}$ in (5) or are modded out when we pass to polarisation vectors. As a result,

$$
\mathrm{i} \cdot \mathrm{i} \rightarrow f^{4} \mathbf{i} \cdot \mathbf{i}, \quad \ddot{\mathbf{l}} \wedge \mathrm{i} \rightarrow\left(f^{2} \ddot{\mathbf{l}}+\alpha_{3} \mathbf{i}\right) \wedge\left(f^{3} \mathbf{i}\right)=f^{5} \mathbf{i} \wedge \ddot{\mathbf{l}}
$$

We find that

$$
K^{a b} \rightarrow f K^{a b}
$$

and so (5) provides a reparametrisation invariant transport law.

3. Inner products between polarization vectors are preserved. This follows from antisymmetry of $K$.

A point worth noting is that $K$ transport involves the second derivative $\ddot{\mathbf{l}}$ of the tangent vector $\mathbf{l}$. This is quite unlike Fermi transport which only involves the first derivative $\dot{\mathbf{t}}$ of the tangent vector. We will see below that this is an unavoidable consequence of the geometry of null vectors.

Unlike Fermi transport, the $K$ transport law does not have a smooth limit as the null curve becomes a geodesic. This will be discussed in the concluding section. 
Note that we are not claiming to transport all vectors along $\mathcal{N}$ in a geometrically natural manner. Our rule is only meant for polarisation vectors, i.e. equivalence classes of vectors perpendicular to the tangent vector.

\section{Comparing Polarisation vectors on distinct Null vectors}

We will now show that the transport rule (5) originates naturally from the geometry of null directions, just as the Fermi transport rule (1) derives from the geometry of timelike directions 《4. If we parallel transport the tangent vector $\mathbf{l}_{p^{\prime}}$ along $\mathcal{N}$ from $p^{\prime}$ to $p$, we find that for non-geodesic curves, the parallel transported tangent vector does not agree with the local tangent vector $\mathbf{l}_{p}$. The parallel tansport of a vector orthogonal to the tangent vector at $p^{\prime}$ is not in general orthogonal to the local tangent vector $\mathbf{l}_{p}$, but to the parallel transported tangent vector. We need to find a way to compare vectors orthogonal to two distinct null vectors. We show below that this can be done in a geometrically natural manner for polarisation vectors.

Let $M$ be a four dimensional vector space with Lorentzian metric $(\eta)$ of signature $(+,-,-,-)$. ( $M$ is a model for $T_{p} \mathcal{M}$, the tangent space at the point $p$ of $\mathcal{M}$.) The set of future pointing $\left(l^{0}>0\right)$, null $\left(l^{a} l^{b} \eta_{a b}=0\right)$ vectors in $M$ forms the future light cone and the set of null directions (defined as future pointing null vectors modulo extent [6]) is a sphere. We will sometimes refer to this sphere as the celestial sphere or the sky (although, strictly speaking, this terminology should be reserved for past pointing null directions).

If $L$ is a null direction and $\mathbf{l}$ a null vector belonging to $L$, we define $H_{L}$ to be the space of vectors $v^{a}$ in $M$ orthogonal to $L, v^{a} l_{a}=0$. $H_{L}$ also includes $L$. We define a polarisation vector $\mathbf{p}_{L}$ to be an equivalence class of vectors in $H_{L}$ differing by an arbitrary multiple of $\mathbf{l}\left(v^{a} \sim v^{a}+\lambda l^{a} \lambda\right.$ arbitrary). The vector space of polarisation vectors defined by $L$ is written $P_{L}$.

Given two distinct null directions $L_{1}$ and $L_{2}$ and a polarisation vector $\mathbf{p}_{1} \in P_{L_{1}}$, there is a geometrically natural choice of a polarisation vector from $P_{L_{2}}$. We pick from $\mathbf{p}_{1}$ the unique element $\mathbf{w} \in H_{L_{1}}$ which is orthogonal to $L_{2}$ and define $\mathbf{p}_{2}$ as the class $\mathbf{w}+\lambda_{2} \mathbf{l}_{2}$ containing the vector $\mathbf{w}$. More explicitly, pick any $\boldsymbol{v}_{\mathbf{1}} \in P_{L_{1}}$. Requiring that

$$
\left(\mathbf{v}_{1}+\lambda_{1} \mathbf{l}_{1}\right) \cdot \mathbf{l}_{2}=0
$$


uniquely fixes $\lambda_{1}$ :

$$
\lambda_{1}=-\frac{\mathbf{v}_{1} \cdot \mathbf{l}_{2}}{\mathbf{l}_{1} \cdot \mathbf{l}_{2}}
$$

which is well defined, since $\mathbf{l}_{1} \cdot \mathbf{l}_{2}>0$, for $\mathbf{l}_{1}$ and $\mathbf{l}_{2}$ distinct. We write

$$
\mathbf{w}=\mathbf{v}_{1}-\frac{\mathbf{v}_{1} \cdot \mathbf{l}_{2}}{\mathbf{l}_{1} \cdot \mathbf{l}_{2}} \mathbf{l}_{1}
$$

and define $\mathbf{p}_{2}$ to be the equivalence class $\mathbf{w}+\lambda_{2} \mathbf{l}_{2}$ containing $\mathbf{w}$ :

$$
\mathbf{v}_{2}=\mathbf{v}_{1}-\frac{\mathbf{v}_{1} \cdot \mathbf{l}_{2}}{\mathbf{l}_{1} \cdot \mathbf{l}_{2}} \mathbf{l}_{1}+\lambda_{2} \mathbf{l}_{2}
$$

Let us write $\delta \mathbf{l}_{12}=\mathbf{l}_{2}-\mathbf{l}_{1}$ and note that $\mathbf{l}_{2} \cdot \mathbf{l}_{2}=0=\left(\mathbf{l}_{1}+\delta \mathbf{l}_{12}\right) \cdot\left(\mathbf{l}_{1}+\delta \mathbf{l}_{12}\right)=2 \delta \mathbf{l}_{12} \cdot \mathbf{l}_{1}+\delta \mathbf{l}_{12} \cdot \delta \mathbf{l}_{12}$ implies

$$
\mathbf{l}_{1} \cdot \delta \mathbf{l}_{12}=-\frac{1}{2} \delta \mathbf{l}_{12} \cdot \delta \mathbf{l}_{12}
$$

We can now write

$$
\mathbf{v}_{2}=\mathbf{v}_{1}-\frac{2 \mathbf{v}_{1} \cdot \delta \mathbf{l}_{12}}{\delta \mathbf{l}_{12} \cdot \delta \mathbf{l}_{12}} \mathbf{l}_{1}+\lambda_{2} \mathbf{l}_{2}
$$

and by suitable choice of $\lambda_{2}$

$$
\mathbf{v}_{2}=\mathbf{v}_{1}-\frac{2\left(\mathbf{v}_{1} \cdot \delta \mathbf{l}_{12}\right) \delta \mathbf{l}_{12}}{\left(\delta \mathbf{l}_{12} \cdot \delta \mathbf{l}_{12}\right)}
$$

This reverses the $\delta \mathbf{l}_{12}$ component of $\mathbf{v}_{1}$ and leaves the component of $\mathbf{v}_{1}$ which is orthogonal to $\delta \mathbf{l}_{12}$ unchanged. The rule which associates polarisation vectors in $P_{1}$ to polarization vectors in $P_{2}$ reverses orientation and therefore cannot be continuously deformed to the identity. This can be seen quite clearly going to a frame in which $\mathbf{l}_{1}$ represents a light ray going in the positive $z$ direction and $\mathbf{l}_{2}$ a light ray going in the negative $z$ direction. Polarization vectors of $\mathbf{l}_{1}$ and $\mathbf{l}_{2}$ can then be identified with the $x-y$ plane but with opposite orientations.

The rule for identifying $P_{1}$ and $P_{2}$ can also be stated succinctly as follows. Consider the two dimensional subspace of $M$ orthogonal to both $\mathbf{l}_{1}$ and $\mathbf{l}_{2}$. The projector on to this subspace is $h_{a b}=\eta_{a b}-\left(\mathbf{l}_{1} \cdot \mathbf{l}_{2}\right)^{-1}\left(l_{1 a} l_{2 b}+l_{2 a} l_{1 b}\right)$. This subspace can be identified with both $P_{1}$ and $P_{2}$ (by taking its elements to represent classes) and this gives an identification of $P_{1}$ with $P_{2}$. As the example of the last paragraph shows, the identification reverses orientation. The natural volume form $\epsilon_{a b}:=\left(\mathbf{l}_{1} \cdot \mathbf{l}_{2}\right)^{-1} \epsilon_{a b c d} l_{1}^{c} l_{2}^{d}$ on the two dimensional subspace reverses sign when $\mathbf{l}_{1}$ and $\mathbf{l}_{2}$ are interchanged.

Since the map from $P_{1}$ to $P_{2}$ cannot be continuously deformed to the identity, it does not have a smooth limit as $L_{2}$ tends to $L_{1}$. Such a smooth limit would be necessary 
to define transport along a smooth null curve. However, if we repeat the process by considering three null directions $L_{1}, L_{2}, L_{3}$ and go from $P_{1}$ to $P_{2}$ and $P_{2}$ to $P_{3}$ using the rule (16) twice, we get an orientation preserving map from $P_{1}$ to $P_{3}$. This map does have a smooth limit as $P_{1}, P_{2}$ and $P_{3}$ approach each other. It is intuitively clear that since we need three null directions (rather than two) to take a smooth limit, the transport law we derive for null vectors will depend on $\mathbf{1}, \mathbf{i}$ and $\ddot{\mathbf{i}}$, in contrast to the Fermi transport law, which only depends on $\mathbf{t}$ and $\dot{\mathbf{t}}$. Writing $\mathbf{l}_{1}, \mathbf{l}_{2}, \mathbf{l}_{3}$ for elements of $L_{1}, L_{2}, L_{3}$ and $\delta \mathbf{l}_{23}=\left(\mathbf{l}_{2}-\mathbf{l}_{3}\right)$ we find as in (16),

$$
\mathbf{v}_{3}=\mathbf{v}_{2}-\frac{2 \mathbf{v}_{2} \cdot \delta \mathbf{l}_{23}}{\delta \mathbf{l}_{23} \cdot \delta \mathbf{l}_{23}} \delta \mathbf{l}_{23}
$$

using (16) we arrive at the following expression for $\mathbf{v}_{3}-\mathbf{v}_{1}$

$$
\frac{4\left(\mathbf{v}_{1} \cdot \delta \mathbf{l}_{12}\right)\left(\delta \mathbf{l}_{12} \cdot \delta \mathbf{l}_{23}\right) \delta \mathbf{l}_{23}-2\left(\mathbf{v}_{1} \cdot \delta \mathbf{l}_{12}\right)\left(\delta \mathbf{l}_{23} \cdot \delta \mathbf{l}_{23}\right) \delta \mathbf{l}_{12}-2\left(\mathbf{v}_{1} \cdot \delta \mathbf{l}_{23}\right)\left(\delta \mathbf{l}_{12} \cdot \delta \mathbf{l}_{12}\right) \delta \mathbf{l}_{23}}{\left(\delta \mathbf{l}_{12} \cdot \delta \mathbf{l}_{12}\right)\left(\delta \mathbf{l}_{23} \cdot \delta \mathbf{l}_{23}\right)} .
$$

We can now take the limit as the three null directions approach each other. Let $\mathbf{l}(\tau)$ be the tangent vector of a smooth null curve. We expand $\mathbf{l}(\tau)$ in a Taylor series.

$$
\begin{aligned}
& \mathbf{l}_{1}=\mathbf{l}(\tau-\Delta \tau)=\mathbf{l}(\tau)-\mathbf{i}(\tau) \Delta \tau+1 / 2 \ddot{\mathbf{l}}(\tau)(\Delta \tau)^{2}+\ldots \\
& \mathbf{l}_{2}=\mathbf{l}(\tau) \\
& \mathbf{l}_{3}=\mathbf{l}(\tau+\Delta \tau)=\mathbf{l}(\tau)+\tau \mathbf{i}(\tau) \Delta+1 / 2 \ddot{\mathbf{l}}(\Delta \tau)^{2}+\ldots
\end{aligned}
$$

where the ellipsis stand for higher order terms than we need. As a result,

$$
\begin{aligned}
\delta \mathbf{l}_{12} & =\mathrm{i}(\tau) \Delta \tau-1 / 2 \ddot{\mathbf{l}}(\Delta \tau)^{2}+\ldots \\
\delta \mathbf{l}_{23} & =\mathrm{i}(\tau) \Delta \tau+1 / 2 \ddot{\mathbf{l}}(\Delta \tau)^{2}+\ldots
\end{aligned}
$$

The leading term in the denominator of (18) is of order $(\Delta \tau)^{4}$ :

$$
(\dot{\mathbf{l}}(\tau) \cdot \mathbf{i}(\tau))(\Delta \tau)^{4}
$$

In the numerator the term of order $(\Delta \tau)^{4}$ is

$$
\left[4\left(\mathbf{v}_{1} \cdot \mathbf{i}\right)(\mathbf{i} \cdot \mathbf{i}) \mathbf{i}-2\left(\mathbf{v}_{1} \cdot \mathbf{i}\right)(\mathbf{i} \cdot \mathbf{i}) \mathbf{i}-2\left(\mathbf{v}_{1} \cdot \mathbf{i}\right)(\mathbf{i} \cdot \mathbf{i}) \mathbf{i}\right](\Delta \tau)^{4}
$$

which vanishes. The first non vanishing term is of order $(\Delta \tau)^{5}$. After some straight forward algebra, we evaluate $\left(\mathbf{v}_{3}-\mathbf{v}_{1}\right) /(2 \Delta \tau)$ and find that the limit $\Delta \tau \rightarrow 0$ exists and yields the $K$ transport law (5) of section II. 
As the reader may have noticed, the entire discussion of this section depends only on the conformal metric and not the metric itself. The definition of $H_{p}$ and "modding out" by $\mathbf{l}$ are unchanged under conformal transformations and so is the rule (16) for comparing polarisation vectors between fibres. One may therefore expect that the $K$ transport rule is conformally invariant. It is easily checked that it is. The $K$ transport of a vector using a conformally rescaled metric only results in trivial rescalings of the polarisation vector (to be expected because parallel transport preserves the norm). Under conformal transformations there is no change in the direction of polarisation of the $K$ transported vector.

\section{Spinorial formulation}

As one might expect, the discussion of the last section can be formulated quite naturally in terms of spinors [6]. Let $\left(V, \epsilon_{A B}\right)$ be a complex 2-dimensional vector space endowed with an antisymmetric non-degenerate tensor $\epsilon_{A B}$. Elements of $V$ are written $\xi^{A}$. The complex conjugate of $\xi^{A}$ is written $\bar{\xi}^{A^{\prime}}$, with $A^{\prime}$ a "primed" or "dotted" spinor index. A pair $A A^{\prime}$ of spinor indices can be converted into a vector index $a$ by using the standard correspondence between vectors and spinors:

$$
v^{a}=\sigma_{A A^{\prime}}^{a} v^{A A^{\prime}},
$$

The components of $\sigma^{a}$ are $\sigma^{0}=I, \sigma^{1}=\sigma^{x}, \sigma^{2}=\sigma^{y}, \sigma^{3}=\sigma^{z}$, and $I$ is the $2 \times 2$ identity matrix and $\left(\sigma^{x}, \sigma^{y}, \sigma^{z}\right)$ are the standard Pauli matrices. We will write such relations (26) as $v^{a} \rightleftharpoons v^{A A^{\prime}}$. The spinor $\xi^{A}$ defines a future pointing null vector $\mathbf{l}^{a} \rightleftharpoons \bar{\xi}^{A^{\prime}} \xi^{A}$. Altering $\xi^{A}$ by a phase does not alter the vector all and multiplying $\xi^{A}$ by a real number alters the extent of the null vector, but not its direction.

Define the following equivalence relation on (non-zero elements of) $V$ :

$$
\xi^{A} \sim \alpha \xi^{A}
$$

where $\alpha$ is any non zero complex number. The set of equivalence classes form a sphere $S^{2}$, which is the set of future pointing null directions - the sky of the previous section. Non zero elements of $V$ form a fibre bundle with the base equal to $S^{2}$ and the fibre isomorphic to the set of non zero complex numbers. The phase of this non-zero complex number determines a "flag plane" [6] or polarisation direction. This is easily seen as follows. Let 
$L_{1}$ be a point on $S^{2}$ and $\xi_{1}^{A}$ a point on the fibre over $L_{1}$. The null vector $l^{a} \rightleftharpoons \bar{\xi}_{1}^{A^{\prime}} \xi_{1}^{A}$ belongs to the null direction $L_{1}$. Let us pick an arbitrary spinor $\xi_{2}$, distinct from $\xi_{1}$, so that $\xi_{2 A} \xi_{1}^{A}$ is nonzero. One can always multiply it by a suitable complex number so that $\xi_{1}^{A} \cdot \xi_{2 A}=1$. The space-like unit vector

$$
v^{a} \rightleftharpoons \frac{1}{\sqrt{2}}\left(\bar{\xi}_{1}^{A^{\prime}} \xi_{2}^{A}+\bar{\xi}_{2}^{A^{\prime}} \xi_{1}^{A}\right)
$$

is clearly orthogonal to $l_{1 a} \rightleftharpoons \bar{\xi}_{1}^{A^{\prime}} \xi_{1}^{A}$

$$
l_{1 a} v^{a}=\frac{1}{\sqrt{2}} \bar{\xi}_{1 A^{\prime}} \xi_{1 A}\left(\bar{\xi}_{1}^{A^{\prime}} \xi_{2}^{A}+\bar{\xi}_{2}^{A^{\prime}} \xi_{1}^{A}\right)=0
$$

A different choiceof $\xi_{2}$, obtained by adding a multiple of $\xi_{1}$ to it, only changes the vector $v^{a}$ in (28) by a multiple of $l_{1}^{a}$. Thus $\xi_{1}^{A}$ determines an equivalence class of unit vectors $v^{a}$ orthogonal to $l_{a}$, i.e., a unit polarization vector. As can be easily verified, altering $\xi_{1}^{A}$ by a phase $e^{i \theta}$ leads to a rotation of the polarization vector by an angle $2 \theta$. Thus, $\xi_{1}^{A}$ and $-\xi_{1}^{A}$ define the same polarization vector. The correspondence is two to one. We thus have a map from the fibre $\mathcal{F}(L)$ over a null direction $L$ to the unit circle of polarization vectors defined by $L$. Our discussion now will be entirely on the spinor bundle.

Given two distinct points $L_{1}$ and $L_{2}$ on the base and a point $\xi_{1}^{A}$ on the fibre $\mathcal{F}\left(L_{1}\right)$ over $L_{1}$, there is a natural way to pick a point $\xi_{2}^{A}$ the $\mathcal{F}\left(L_{2}\right)$ fibre over $L_{2}$. We pick the unique point $\xi_{2}^{A}$ which satisfies

$$
\xi_{1}^{A} \xi_{2 A}=1
$$

(This choice when translated into vectors agrees with the discussion of section IV). The rule (30) is well defined only if $L_{1}$ and $L_{2}$ are distinct points. If $\xi_{1}^{A}$ is altered by a phase $\xi_{1}^{A} \rightarrow e^{i \theta} \xi_{1}^{A}, \xi_{2}^{A}$ picks up the opposite phase: $\xi_{2}^{A} \rightarrow e^{-i \theta} \xi_{2}^{A}$. Thus, rule (30) maps a circle winding in the anticlockwise sense to a circle winding in the clockwise sense. The map defined by (30) from $\mathcal{F}\left(L_{1}\right)$ to $\mathcal{F}\left(L_{2}\right)$ cannot be continuously deformed to the identity and the rule (30) does not admit a smooth limit as $L_{2}$ approaches $L_{1}$.

As in Section 4, we can solve this problem by considering three points, $L_{1}, L_{2}, L_{3}$ on $S^{2}$. Given $\xi_{1}^{A} \in \mathcal{F}\left(L_{1}\right)$ we pick $\xi_{2}^{A}$ from $\mathcal{F}\left(L_{2}\right)$ accordingly to the rule (30) and repeat the process to pick $\xi_{3}^{A}$ from $L_{3}$ using $\xi_{2}^{A} \xi_{3 A}=1$. The map from $L_{1}$ to $L_{3}$ does admit a smooth limit as $L_{1}, L_{2}$ and $L_{3}$ approach each other. We will use this below to derive the spinorial form of the transport law (5). If $L_{1}, L_{2}$ and $L_{3}$ are three distinct null directions, the map from $L_{1}$ to $L_{3}$ (via $L_{2}$ ) does depend on $L_{2}$. If a different choice $L_{2}^{\prime}$ is made, one can check 
that the point $\xi_{3}$ on $\mathcal{F}\left(L_{3}\right)$ determined by $\xi_{1}$ is multiplied by a complex number $\chi$, where

$$
\chi=\frac{\left(\xi_{1}^{A} \xi_{2^{\prime} A}\right)\left(\xi_{2}^{B} \xi_{3 B}\right)}{\left(\xi_{1}^{D} \xi_{2 D}\right)\left(\xi_{2^{\prime}}^{C} \xi_{3 C}\right)} .
$$

$\chi$ depends only on the four null directions $L_{1}, L_{2}, L_{3}$ and $L_{2^{\prime}}$ and not on the representatives chosen from each fibre. $\chi$ is called the cross ratio [6] of these four null directions. The fact that the map from $L_{1}$ to $L_{3}$ does depend on $L_{2}$, ( $\chi$ is not the identity) shows that the discrete rule for comparing fibres over distinct null directions is not integrable.

We will now take the continuous limit of the discrete rule and recover the transport law (5) in spinorial language. We are given a curve $L(\tau)$ of null directions and a point $\xi(0)$ on the fibre over $L(0)$. What we seek is a geometrically natural "lift" of this curve i.e. we need to find $\xi(\tau)$ so that $\xi(\tau) \in \mathcal{F}(L(\tau))$.

Fix a spin frame $\left(\dot{i}^{A}, o^{A}\right)\left(i^{A} o_{A}=1\right)$ and write $\xi(\tau)=\gamma(\tau)\left(i^{A}+z(\tau) o^{A}\right) . \quad z$ is a stereographic coordinate on the set of null directions and $\gamma$ is a coordinate on the fibre. The problem now is: give a smooth curve $z(\tau)$, determine $\gamma(\tau)$ using the rule (16). We expand $z(\tau)$ in a Taylor series and write

$$
\begin{aligned}
z_{1} & =z(\tau-\Delta \tau)=z(\tau)-\dot{z} \Delta \tau+\frac{1}{2} \ddot{z}(\Delta \tau)^{2}+\ldots \\
z_{2} & =z(\tau) \\
z_{3} & =z(\tau+\Delta \tau)=z(\tau)+\dot{z} \Delta \tau+\frac{1}{2} \ddot{z}(\Delta \tau)^{2} .
\end{aligned}
$$

Evidently,

$$
\begin{aligned}
& \xi_{1}=\xi(\tau+\Delta \tau)=\gamma(\tau-\Delta \tau)\left(i^{A}+z(\tau-\Delta \tau) o^{A}\right) \\
& \xi_{2}=\xi(\tau)=\gamma(\tau)\left(i^{A}+z(\tau) o^{A}\right) \\
& \xi_{3}=\xi(\tau+\Delta \tau)=\gamma(\tau \Delta+\tau)\left(i^{A}+z(\tau+\Delta \tau) o^{A}\right) .
\end{aligned}
$$

Using the rule (16) for determining $\xi_{2}$ and $\xi_{3}$ we find from $\xi_{1}^{A} \xi_{2 A}=\xi_{2}^{A} \xi_{3 A}=1$,

$$
\begin{aligned}
& \gamma(\tau) \gamma(\tau-\Delta \tau)(z(\tau)-z(\tau-\Delta \tau)=1 \\
& \gamma(\tau) \gamma(\tau+\Delta \tau)(z(\tau+\Delta \tau)-z(\tau)=1
\end{aligned}
$$

We eliminate $\gamma(\tau)$ from these equations and find using the Taylor expansion for $z(\tau)$

$$
\frac{\gamma(\tau+\Delta \tau)-\gamma(\tau-\Delta \tau)}{\gamma(\tau-\Delta \tau)}=\frac{\left(\dot{z} \Delta \tau-1 / 2 \ddot{z}(\Delta \tau)^{2}+\ldots\right)-\left(\dot{z} \Delta \tau+1 / 2 \ddot{z}(\Delta \tau)^{2}+\ldots\right)}{(\dot{z} \Delta \tau+\ldots)}
$$


Evaluating

$$
\frac{\gamma(\tau+\Delta \tau)-\gamma(\tau-\Delta \tau)}{\gamma(\tau-\Delta \tau) 2 \Delta \tau}
$$

we find in the limit $\Delta \tau \rightarrow 0$

$$
\gamma^{-1} \dot{\gamma}=(-1 / 2) \ddot{z} / \dot{z}
$$

which is the transport law (5) in spinorial form. Its this form, it is apparent that (41) can be integrated to yield

$$
\gamma(\tau)=\gamma(0) \sqrt{(\dot{z}(0) / \dot{z}(\tau))}
$$

Although the discrete rule (30) for comparing points on distinct fibres is not integrable, its continuous limit (the K-transport rule) is. This is a feature of $\mathrm{K}$-transport which is not shared by Fermi transport. It is interesting that the discrete rule is not integrable, while the continuum limit is. This indicates that as the four null directions $L_{1}, L_{2}, L_{2}^{\prime}, L_{3}$ tend to each other, the phase discrepancy between alternative paths vanishes sufficiently fast that there is none left in the continuum limit.

\section{Relation to the Geometric Phase in two state quantum mechanics}

The discussion so far has been entirely Lorentz invariant. In particular the last section has been in the language of $S L(2, \mathscr{C})$ spinors. We will now attempt to make contact with the notion of the Geometric Phase in quantum mechanics [7]. The motivation is as follows. One can think of the spinors of the previous section as describing the state vectors of a two state quantum mechanical system. The equivalence relation (27) defining the fibres is precisely the one which takes one from Hilbert space to the space of physical states (ray space). The relation (30) associates a member $\xi_{2}$ of the fibre over the point $L_{2}$ is associated with a particular member $\xi_{1}$ of the fibre over $L_{1}$. In quantum mechanics, there is a notion of two state vectors, corresponding to different rays, being "in phase", which leads to the geometric phase. It is natural, therefore, to ask whether the relation (30) has an analogue in quantum mechanics. At first sight, there is an obstacle. The relation (30) breaks down when the two null vectors $L_{1}$ and $L_{2}$ are coincident. The geometric phase convention in quantum mechanics breaks down when the two rays are orthogonal. Nevertheless, there is a close correspondence which is developed in this section. In order to do this we need to reduce the structure group from $S L(2, \mathbb{C})$ to $S U(2)$. The $S L(2, \mathbb{C})$ 
invariant structures that we described in the last section will now be described in terms of $S U(2)$ spinors.

In order to break the structure group down from $S L(2, \mathbb{C})$ to $S U(2)$, we introduce on the two complex dimensional vector space $\left(V, \epsilon_{A B}\right)$ an additional structure: a positive definite Hermitian inner product $G_{A^{\prime} A}$. One can think of $G_{A A^{\prime}}$ as the spinor corresponding to a timelike four vector. Thus making a choice of $G$ is like making a choice of the fourvelocity of a frame of reference, which still leaves freedom to make spatial rotations. The group of transformations that preserves both $\epsilon_{A B}$ and $G_{A A^{\prime}}$ is $S U(2)$. By choice of spin frame $\left(\iota^{A}, o^{A}\right)$ we can arrange that

$$
G_{A^{\prime} A}=\iota_{A} \iota_{A^{\prime}}+o_{A} o_{A^{\prime}}
$$

and use $G_{A^{\prime}}{ }^{A}$ to define a $\dagger$ operation taking a spinor $\xi^{A}$ to a new spinor $\xi^{\dagger}$ transforming in the same way.

$$
\xi^{\dagger A}:=\bar{\xi}^{A^{\prime}} G_{A^{\prime}}{ }^{A}
$$

We will sometimes use Dirac notation $\mid \xi>$ for the element $\xi^{A}$ of $V$ and $<\xi \mid$ for the element $\xi_{A}^{\dagger}$ of $V^{*}$ (the dual of $V$ ). Note that $<\xi \mid$ is not $\xi_{A}$, for $<\xi \mid \xi>$ is positive definite whereas $\xi_{A} \xi^{A}$ vanishes. It is easily checked that $\xi_{A}^{\dagger \dagger}=-\xi_{A}$ and that $\xi^{\dagger}$ is orthogonal to $\xi, i . e<\xi^{\dagger} \mid \xi>=0$. The action of $\dagger$ on the sphere of null directions (the sky) is easy to visualise. By explicit computation are sees that $\iota^{\dagger}=o, o^{\dagger}=-\iota$ and so, if $\xi^{A}=i^{A}+z o^{A}$, $\xi^{\dagger A}=-\bar{z} i^{A}+o^{A}$, so $\dagger$ sends each point on the sky to its antipode. The subgroup of $S L(2, \mathscr{C})$ which preserves the relation of antipodality is $S U(2)$, which acts on the sky by rotations.

We now identify the $S U(2)$ spinors with state vectors of a two state system and the sky with the corresponding ray space, which is a sphere. (Historically, this arose in the context of polarised light, which can be represented by a pair of complex numbers, and the sphere was discovered by Poincaré after whom it is named. The definition that two states are in phase when their inner product is real and positive was proposed by Pancharatnam [7]). The rule $\xi_{1}^{A} \cdot \xi_{2 A}=1$ for comparing points on distinct fibres can be rewritten as

$$
\xi_{1 A}^{\dagger \dagger} \xi_{2}^{A}=<\xi_{1}^{\dagger} \mid \xi_{2}>=1
$$

i.e, we require that $\mid \xi_{2}>$ be in phase with $\mid \xi_{1}^{\dagger}>$. (We are not concerned here with the modulus of the complex number $\left\langle\xi_{1}^{\dagger}\right| \xi_{2}>$, but only its phase. ) The rule (45) is well defined if $\xi_{1}$ and $\xi_{2}$ are on distinct fibres, or, equivalently, if $\xi_{2}$ and $\xi_{1}^{\dagger}$ are not antipodal. 
As in the case of vectors, the rule (30) for passing from the fibre $\mathcal{F}\left(L_{1}\right)$ over $L_{1}$ to the fibre $\mathcal{F}\left(L_{3}\right)$ over $L_{3}$ (via $L_{2}$ ) will depend on $L_{2}$. This dependence is captured by the cross ratio (31). The phase of the complex number (31) is a measure of the non-integrable nature of the rule (30) for comparing points on distinct fibres. We can rewrite this quantity as the phase of the complex number

$$
<\xi_{1}\left|\xi_{2}^{\dagger}><\xi_{2}^{\dagger}\right| \xi_{3}><\xi_{3}\left|\xi_{2^{\prime}}^{\dagger}><\xi_{2^{\prime}}^{\dagger}\right| \xi_{1}>
$$

which has a simple geometric interpretation. Consider the four points $L_{1}, \tilde{L_{2}}, \tilde{L_{2^{\prime}}}$ and $L_{3}$ on the celestial sphere, where $\tilde{L_{2}}$ and $\tilde{L_{2^{\prime}}}$ are points antipodal to $L_{2}$ and $L_{2^{\prime}}$ respectively. The phase of $\chi$ has an interpretation which is well known in the Geometric phase literature [7]: it is equal to half the solid angle subtended at the center of the sphere by the geodesic rectangle $L_{1}, \tilde{L_{2}}, \tilde{L_{3}}, \tilde{L_{2^{\prime}}}, L_{1}$. It follows that the change in the plane of polarisation in following the route $L_{1}, \tilde{L_{2}}, L_{3}, \tilde{L_{2^{\prime}}}, L_{1}$ is equal to the solid angle subtended by this rectangle. Although this change in the plane of polarisation has been computed in language pertaining to a given frame of reference, it is of course Lorentz invariant, from the earlier discussion.

\section{Concluding Discussion}

We have presented a transport law (5) which is the replacement for Fermi transport in the case of null curves. We have also shown how this transport law arises naturally from the geometry of null vectors. The $K$ transport law has a natural description in terms of $S L(2, \mathscr{C})$ spinors. This description also brings out close analogies with the geometric phase, once it is specialised to $S U(2)$ spinors by choosing a timelike observer. In the rest of this section we compare the $K$ transport law and Fermi transport.

The main difference between Fermi transport and $K$ transport is due to the difference between the geometry of timelike directions and the geometry of null directions. The set of timelike directions can be identified with a time-like 3-hyperboloid, whose isometry group is the entire Lorentz group. In contrast, the null directions are identified with a 2 -sphere and the Lorentz group acts on the sphere by conformal transformations. There is consequently no Lorentz invariant meaning to the statment that two null directions are "near" each other. By a suitable Lorentz transformation, any two distinct null directions can be made antipodal. As a result, there is no Lorentz and reparametrization invariant measure of the "acceleration" of a null curve. If the direction of the tangent vector of a 
null curve changes "slightly" in one Lorentz frame, this deviation can be made as large as one pleases in some other Lorentz frame.

Fermi transport reduces smoothly to parallel transport when the timelike curve becomes a timelike geodesic. In contrast, the transition from null curves to null geodesics is a singular one. This is reflected in the absence of a smooth limit for $K$ transport. As an example, let $\mathcal{M}$ be Minkowski space with standard $(t, x, y, z)$ Cartesian co-ordinates and consider the null curve: $x=R \cos (\Omega \tau), y=R \sin (\Omega \tau), z=\tau, t=\left(\sqrt{1+R^{2} \Omega^{2}}\right) \tau$, where $R$ and $\Omega$ are constants. This curve describes a particle moving at the speed of light along a helical path.

The $K$ tensor for this null curve is easily worked out to be $K=\Omega d x \wedge d y$ and is independent of $R$. The transport rule simply says that the polarisation vector rotates about the $z$-axis with angular velocity $\Omega$. This is of course the angular velocity of the frame made up by the spatial tangent and the normal, i.e the Serret-Frenet frame.

In the limit that $R$ tends to 0 with $\Omega$ finite, the null curve does become a geodesic curve. However, $K$-transport does not reduce to parallel transport Thus, the limit of $K$ transport to null geodesics is not smooth. In fact, one can easily do the above Minkowskispace calculation for motion at the speed of light along a general smooth space curve whose spatial curvature nowhere vanishes. We can choose the length of the curve as a parameter, and choose polarisation vectors to be purely spatial. As in the case of the helix, one finds that $K$ transport reduces to Serret-Frenet transport, which is an integrable rule. One other way to check this is to compute the rate of change of the cosine of the angle made by the transported vector $\mathbf{v}$ with the acceleration vector $\mathbf{i}$. A straightforward calculation using $K$-transport shows that

$$
\frac{d}{d \tau}\left(\frac{\mathbf{v} \cdot \mathbf{i}}{\sqrt{\mathbf{i} \cdot \mathbf{i}}}\right)=0
$$

Since the acceleration vector vanishes for geodesics, the rule must and does become illdefined in the geodesic limit, as does Serret-Frenet transport.

Although the discrete rule given above for comparing polarisation vectors on distinct fibres is not integrable, the limit of this rule for smooth curves $i s$ integrable. This is a feature of the $K$ transport rule, which is different from Fermi transport. We note that if the rule had not been integrable in the continuous limit, we would have been able to define a two-form on the base space (the sky) whose integral around a closed curve would give the total rotation on traversing that curve. But, as is well known, there is no Lorentz 
invariant notion of area on the space of null vectors, except the one which assigns zero to each such area. With hindsight, the integrability is consistent with, and even forced by, Lorentz invariance.

Finally, we close with the remark that on spinors, $K$ transport is only locally integrable. (42) fixes $\gamma(\tau)$ only up to a sign. This sign is unimportant in discussing the transport of polarisation vectors. However, in transporting spinors along null, non-geodesic curves one can see a non integrable phase of topological origin with values \pm 1 . The result of spinor transport is not affected by continuous deformations of the null curve $\mathcal{N}$ within the class of null, everywhere non-geodesic curves, connecting the end points of $\mathcal{N}$, but is affected by changes in $\mathcal{N}$ which cannot be continuously deformed away. Let $z(\tau)$ be a smooth closed simple (i.e non self-intersecting) curve in the space of null directions, with $\dot{z}(\tau)$ being nowhere zero. The complex number $\dot{z}(\tau)$ encircles the origin once in the complex plane for such a curve. Equation (42) then shows that transporting a spinor once along this curve results in a phase difference of $\pi$, which is in principle observable by interference. Acknowledgments: J.S. thanks V.P. Kattabomman for a discussion on transport rules.

\section{References}

[1] S. Weinberg, "Gravitation and Cosmology", John Wiley and sons, (1972).

[2] C.W. Misner, K.S. Thorne and J.A. Wheeler, Gravitation, W.H. Freeman, NY (1973).

[3] L.D. Landau and E.M. Lifshitz "Classical Theory of Fields", Pergamon Press (1975).

[4] H. Urbantke, Am. J. Phys. 58, 747 (1990).

[5] S.W.Hawking and G.F.R.Ellis " The Large Scale Structure of Space-Time "Cambridge (1973)

[6] R. Penrose and W. Rindler, "Spinors and Space-time", CUP, (1986), Cambridge.

[7] See "Geometric Phases in Physics" Eds. Alfred Shapere and Frank Wilczek, World Scientific, Singapore (1989). 